\title{
TMT Approach to Observatory Software Development Process
}

Hanne Buur, Annapurni Subramaniam, Kim Gillies, Christophe Dumas, Ravinder Bhatia

Thirty Meter Telescope International Observatory, 100 West Walnut St., Suite 300, Pasadena CA, 91124, USA

\begin{abstract}
The purpose of the Observatory Software System (OSW) is to integrate all software and hardware components of the Thirty Meter Telescope (TMT) to enable observations and data capture; thus it is a complex software system that is defined by four principal software subsystems: Common Software (CSW), Executive Software (ESW), Data Management System (DMS) and Science Operations Support System (SOSS), all of which have interdependencies with the observatory control systems and data acquisition systems. Therefore, the software development process and plan must consider dependencies to other subsystems, manage architecture, interfaces and design, manage software scope and complexity, and standardize and optimize use of resources and tools. Additionally, the TMT Observatory Software will largely be developed in India through TMT's workshare relationship with the India TMT Coordination Centre (ITCC) and use of Indian software industry vendors, which adds complexity and challenges to the software development process, communication and coordination of activities and priorities as well as measuring performance and managing quality and risk. The software project management challenge for the TMT OSW is thus a multi-faceted technical, managerial, communications and interpersonal relations challenge. The approach TMT is using to manage this multifaceted challenge is a combination of establishing an effective geographically distributed software team (Integrated Product Team) with strong project management and technical leadership provided by the TMT Project Office (PO) and the ITCC partner to manage plans, process, performance, risk and quality, and to facilitate effective communications; establishing an effective cross-functional software management team composed of stakeholders, OSW leadership and ITCC leadership to manage dependencies and software release plans, technical complexities and change to approved interfaces, architecture, design and tool set, and to facilitate effective communications; adopting an agile-based software development process across the observatory to enable frequent software releases to help mitigate subsystem interdependencies; defining concise scope and work packages for each of the OSW subsystems to facilitate effective outsourcing of software deliverables to the ITCC partner, and to enable performance monitoring and risk management. At this stage, the architecture and high-level design of the software system has been established and reviewed. During construction each subsystem will have a final design phase with reviews, followed by implementation and testing. The results of the TMT approach to the Observatory Software development process will only be preliminary at the time of the submittal of this paper, but it is anticipated that the early results will be a favorable indication of progress.
\end{abstract}

Keywords: Agile process, engagement model, decomposition, traceability, performance tracking

\section{INTRODUCTION}

The TMT will explore the origin of galaxies, reveal the birth and death of stars, probe the turbulent regions surrounding supermassive black holes, and uncover previously hidden details about planets orbiting distant stars, including the possibility of life on these alien worlds. TMT is currently scheduled to achieve first-light in 2027 and start observatory operations soon thereafter.

The Indian partner to the TMT International Observatory (TIO) is the Department of Science and Technology (Government of India), on behalf of which the India TMT Coordination Centre (ITCC) has been assigned several software and hardware responsibilities under the formal TIO workshare agreement, including delivering all TMT Observatory Software per the software requirements, architecture and design defined by the TMT Project Office. The OSW ITCC staff is affiliated with the Indian Institute of Astrophysics, Sarjapur Road, Koramangala II block, Bangalore, India.

As discussed in the abstract, the Observatory Software (OSW) project is a multi-faceted software project management challenge and it has required a multi-pronged approach in order not to significantly delay the OSW schedule and thereby impact several other TMT subsystems. This multi-pronged approach is also a focused method for managing risk. The OSW Team went through a risk analysis phase where the key risks and their associated mitigation strategies were identified. A "roadmap" was developed, which captured and mapped out over time the critical items, risk mitigation 
strategies and decisions that needed to be planned and executed in parallel over the first two quarters of 2016, starting with the formation of two key teams to plan, coordinate and manage activities and challenges as follows.

First, an OSW management team (referred to as the OSW Team) was formed with key staff from the TMT PO and ITCC. The OSW Team started meeting weekly with focused agendas to plan all the OSW activities and make strategic decisions. The critical path for OSW is the development of the Common Software (CSW), which is the infrastructure for the TMT software subsystems, so the OSW Team has primarily focused on getting the CSW final design completed, developing an implementation and test plan, and selecting a qualified vendor.

Second, a TMT Software Stakeholder Working Group (SW Stakeholder WG) was formed with members from all TMT software subsystems and TMT partners involved with software development. The SW Stakeholder WG was officially kicked-off in February 2016 with a review and agreement on the WG charter, which defines the scope, objective, roles and responsibilities, and membership of the WG. The objective of the TMT Software SW Stakeholder WG is to coordinate cross-functional software development activities, resolve technical challenges and enable collaboration amongst all TMT software subsystem teams (SSTs). The team has been meeting monthly since the kick-off with focused agendas, and so far the attendance is high and the meetings have been very productive. The team is already tackling many issues and process improvement initiatives, such as, software licensing issues, CSW prototyping and design refinement, definition of TMT software quality metrics, software interface control database, updates to the TMT Software Design Process, software failure handling, and CSW use case review.

The other critical items and risk mitigation strategies captured in the "roadmap" were: 1) Develop an engagement and resource model for managing the complexities and risks associated with outsourcing of the OSW development effort and a geographically distributed team, and to facilitate effective communication. 2) Implement a vendor selection process that will result in the selection of qualified vendors through best-value contracts to minimize risk. 3) Define a concise CSW scope and fine tune the CSW design through additional prototyping activities and use case definition, to be reviewed and approved by the SW Stakeholder WG. 4) Decompose the CSW features and requirements into a clearly defined set of user stories, and estimate the effort associated with each user story as a basis for an implementation and test plan that will guide the procurement process and the software development activities. 5) Develop a performance tracking method based on the user stories traceable to the technical requirements and the required software features to manage the performance risks associated with outsourcing. 6) Finalize the Agile based TMT Software Development Process in preparation for the software development activities in India.

These critical activities will be further discussed in this paper, except for the CSW design and prototyping activities since the focus is on project management and process. Additionally, an example of the CSW estimation effort based on the user story decomposition will be provided.

\section{TMT SOFTWARE DEVELOPMENT PROCESS}

The TMT Software Development Process (SDP) is based on the Agile software development methodology; it specifically includes frequent iterations and software releases, feature driven development, continuous integration and automated testing aspects. The TMT iteration cycle is shown in Figure 1. It starts with iteration planning where the team decides the scope of work for the upcoming iteration. The scope of work is based on prioritized features defined by their requirements and associated user stories from the user story list (backlog) as well as prioritized issues that need to be resolved. Following the planning session, the software development team enters an internal development phase characterized by daily cycles of detailed design, coding, build, integration and testing. The unit tests are developed in parallel with the functional software as part of the continuous integration and automated test system. At the completion of each iteration the development team releases the tested software to the TMT Software Testing and Integration Lab (STIL) for system integration and further testing, including quality checks, system verification tests, performance tests, etc. The results of the STIL activities are captured in reports that are made available to all stakeholders. The reports are a key input at the iteration review at the end of the iteration cycle, where the team reviews lessons learned and metrics, opportunities for improvements, quality issues identified, etc. The results of the iteration review is then applied to the planning session for the next iteration.

In the TMT SDP, software development iterations are fixed-time development cycles. The standard length of the iteration is 4 weeks to enable the software development activities to align with the TMT-required earned value management as discussed in section 5 below. 


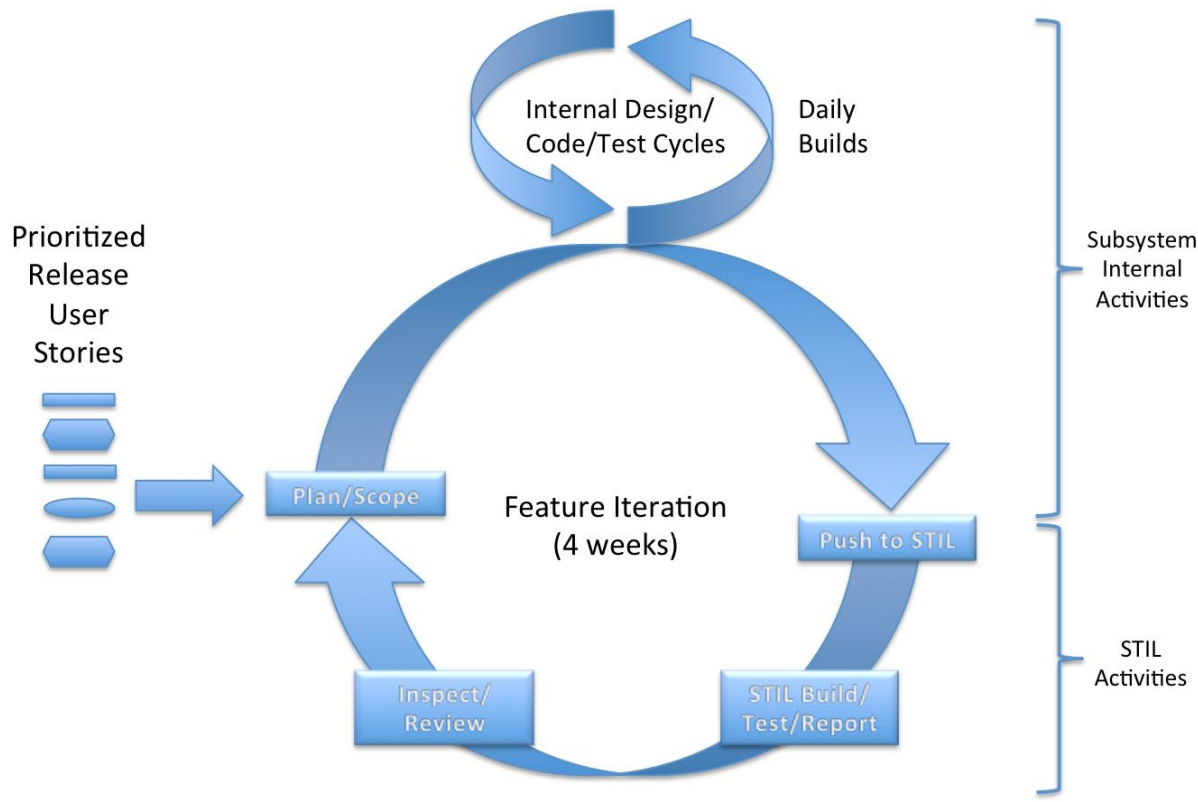

Figure 1. TMT SDP Iteration Development Cycle

Within the TMT project, the subsystem functions to be developed are defined at the concept level and as high-level requirements. The subsystem functions are further decomposed into features, and these are specified by the subsystem technical requirements captured in the subsystem Design Requirements Document (DRD) and Interface Control Document (ICD). Each feature is further decomposed into a set of user stories, which describe what the software will do to implement a requirement. Thus, the DRD and the ICD become the source of the user stories. The software requirements contained in the DRD and ICD documents will be formally traced to user stories for requirements verification. Requirements verification is a critical part of the software deliverable acceptance process as discussed in section 5, so traceability of the successfully tested software deliverables to the user stories and ultimately the requirements and features they implement is being built into the TMT software project management and systems engineering processes. The traceability of software deliverables to user stories, requirements and features is also key to the planned method for tracking software development project performance and to managing cost, schedule and performance risk. The performance tracking method is further discussed in section 5 .

\section{ENGAGEMENT AND RESOURCE MODEL}

The development of Observatory Software (OSW) will be performed in India by pre-qualified software vendors. The vendor selection process will be based on the standard process discussed below with the selection criteria defined by India TMT Coordination Centre (ITCC) and TMT Project Office (TMT PO) as a "best-value" criteria. The selected software vendors will be required to manage the day-to-day activities of their software development staff at their facilities in accordance with the terms of the contract and the scope of work agreed upon for each iteration. The selected software vendors will be required to comply with the TMT Software Systems Engineering processes as defined in the TMT Software Development Process document, the TMT Software Quality Assurance Plan and the TMT Software Configuration Management Plan. They will also be required to work under technical direction from the ITCC and TMT PO OSW Team as well as report status to the TMT Observatory Software Project Manager and the ITCC Observatory Software Work Package Manager. In addition, the selected software vendors will be required to provide contract deliverables to the ITCC and TMT PO OSW Team for review, test and acceptance. The contract deliverables will only be accepted following both ITCC and TMT PO review, test and acceptance, thus ITCC and TMT PO both have a responsibility for the acceptance process per the acceptance criteria specified in the vendor contract. 
The TMT OSW Team has defined an engagement and resource model to manage effectively the software development activities in India, and to mitigate the complexity and risks associated with outsourcing of software development activities and working with geographically distributed teams. The primary risks are ineffective communication and oversight potentially resulting in quality issues, schedule slip and cost creep, and deliverables not meeting the TMT requirements or acceptance criteria. The engagement model and the associated resource model will be applied consistently to all software development activities performed in partnership with Indian software industry partners as follows: There are three partners in the engagement model that will need to have clear roles and responsibilities defined in the context of the engagement model. The three partners must have frequent and open communications which will be facilitated through a three-way OSW Management Team that will meet regularly to discuss all technical and programmatic matters. The OSW Management Team will be led by the TMT OSW Project Manager, the ITCC OSW Work Package Manager and the Indian SW Vendor Program Manager. This management and communications model is represented as an equilateral triangle in Figure 2 below with the OSW Management Team as the focal point for the communication and management of the contracted activities.

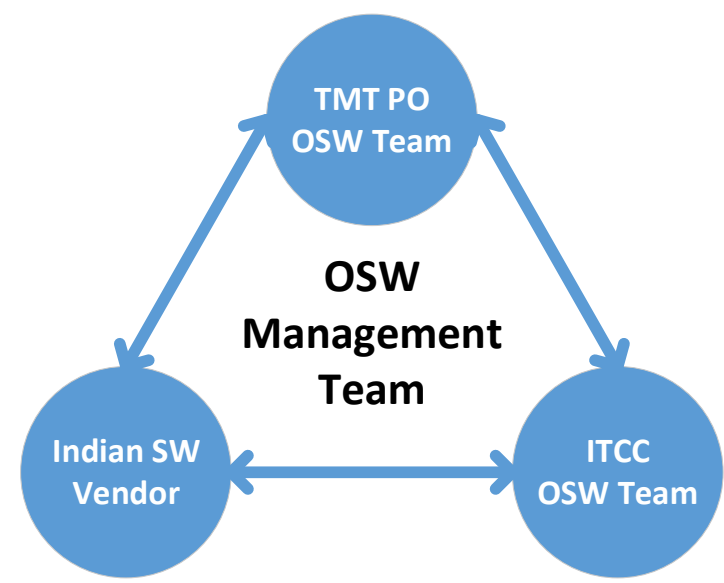

Figure 2. TMT Observatory Software (OSW) management and communications model

The technical leadership of the development activities in India will be managed and coordinated through an Integrated Product Team (IPT), which will be led by the TMT PO OSW Team due to its observatory software experience and software development expertise. As such, the TMT PO OSW Team will provide technical direction and support to the Indian software vendor on a daily basis. The ITCC OSW team does not yet have the necessary domain experience or expertise to lead the software development activities, but it will participate in all IPT activities, including software design, iteration planning and review sessions, software testing, and debugging, which will enable the ITCC OSW Team to develop domain knowledge during the TMT OSW development process. ITCC will be in direct communication with the vendor on a day-to-day basis for immediate technical (within their level of expertise) and programmatic matters. The primary responsibilities of each of the three partners have been clearly defined and captured in the TMT OSW Software Project Management Plan. The responsibilities can be summarized as follows:

- $\quad$ TMT PO OSW Team: Owner of OSW requirements and responsible for requirements flow-down to the Indian software vendor; provides technical leadership, direction and domain expertise; plans scope of each iteration; leads the OSW IPT; co-leads the OSW Management Team; tracks and measures progress to plan; reviews, tests and accepts OSW deliverables

- ITCC OSW Team: Responsible for ITCC procurement process and contract management; participates in OSW IPT; co-leads the OSW Management Team; supports iteration planning; provides daily vendor support; tracks and measures progress per the contract; reviews, tests and accepts OSW deliverables

- Indian Software Vendor: Performs the SW development activities per the contract and the technical direction provided by the TMT PO staff, and delivers required source code; manages vendor software development staff and deliverables per the terms of the contract at the vendor facility; unit and component tests all software before delivery; supports TMT PO and ITCC OSW Teams with iteration planning and iteration reviews; participates in OSW IPT and OSW Management Team 
This engagement and resource model has been embraced by the OSW Team members and officially approved by the TMT project Manager and the Director of ITCC. It represents a shift from the original plan of doing in-house development at ITCC with newly hired and contracted resources, a model that was deemed too risky due to ITCC's limited software development experience. The risk with the new model is the timeliness of the technical leadership being provided by the TMT PO OSW Team; untimely technical direction and feedback may delay the development activities and increase the cost of the effort. The TMT PO OSW Team plans on mitigating that risk through the hire of additional staff who will be responsible for technical support of the Indian software vendor as well as supporting all OSW design, implementation, testing and debugging activities.

\section{VENDOR SELECTION PROCESS}

The OSW procurement process for software development services is owned and managed by the ITCC OSW Team with support from the TMTPO OSW Team. The selection of an industry partner - a technically qualified vendor who can provide software development services per project requirements - will be through a two phase procurement process as follows:

1. Expression of Interest (EoI) phase: A request for Expression of Interest (EoI) is advertised openly and broadly in India; this process is similar to a Request For Information (RFI) process in the US. The EoI responses from vendors are the formally evaluated against a published criteria. The evaluation process also includes face-toface meetings with the vendors at their facilities to gain further insight into their capabilities, facilities, quality, experience and interest. The result is a list of pre-qualified vendors based on their technical and management capabilities, quality system, financial stability and past performance

2. Request for Proposal (RFP) phase: The pre-qualified vendors are invited to submit technical and commercial bids which will be evaluated and used to select a vendor based on a "best-value" criteria to perform a specific statement of work (SOW). The "best-value" criteria is based on meeting technical expertise and experience, cost and schedule requirements

The OSW procurement process will have formal oversight from the ITCC Software Committee, whose membership includes leaders from the Indian Institute of Astrophysics (scientists and administrators) and Inter University Centre for Astronomy and Astrophysics (scientists and administrators) as well as key software subsystem representatives from the TMT PO. The Software Committee also has representation from the senior management team that is developing India's software contribution to the Square Kilometer Array. The Software Committee will ensure that the procurement process has been fairly, openly and consistently executed per the EoI and RFP criteria. Additionally, the administrators will insure that Indian government procurement rules and regulations have been satisfied.

The OSW Team has already completed the first phase of the procurement process (EoI) for the OSW development project. The EoI phase was completed in 3 months, and it resulted in the qualification of three Indian vendors. The EoI phase included face-to-face meetings with the vendors at their facilities. These meetings followed a pre-established agenda defined by the OSW Team: 1) Presentation on relevant experience, technical competence and compatibility, facilities and infrastructure overview, examples of international team experience, and project management competence and experience. 2) Tailored questions to understand better the vendor's experience with the software development life cycle, software systems engineering methods and processes, software quality assurance methods and processes, software configuration management processes and tools, and agile software development processes and tools. 3) Tailored questions related to the submitted EoI response to address any missing information or clarify ambiguous information in the submittal. 4) Tour of the facility to assess their facilities and infrastructure, and to meet with actual software team members to gauge their experience. The face-to-face meetings were very successful and very beneficial to the vendor selection process; they were very focused, productive and interactive.

The OSW Team is now preparing the RFP for the second phase of the procurement process, which will be submitted in June 2016 to the pre-qualified vendors. The scope of this RFP is the CSW development project. The RFP will contain a precise SOW for the CSW scope with predefined milestones based on the desired schedule duration and the required CSW features and their associated acceptance criteria. These milestones will essentially serve as "break-points" in the contract at which time the vendor's deliverables, performance and compliance with TMT processes will be assessed per the contract management process discussed below. The RFP will also include a list of technical requirements and their associated user stories, which will enable the vendors to provide a detailed schedule and cost estimate. The OSW Team will compare that to the CSW effort and cost estimates we have developed based on the set of user stories (see example 
below) to gauge the validity of the commercial bid. The vendors will be required to identify key personnel and their skill sets available to the CSW project in their RFP bid response. They will be required to participate in the frequent meetings as identified in the engagement and resource model, and they will be required to travel to the TMT PO and to ITCC for training and key review meetings. Finally, they will be required to deliver performance and quality metrics per the TMT SDP and the TMT Software Quality Assurance Plan. The plan for accomplishing these requirements must also be included in the RFP bid response, and all will be assessed against the "best-value" criteria. The best-value criteria will be defined as follows:

1. Technical qualification: Technical and programmatic compatibility with the RFP requirements

2. Cost competiveness: This will only apply to vendors that have been identified as "technically qualified" for that specific RFP

The "best-value" approach outlined above is unique to the TMT partner outsourcing agreements in that the primary criterion in the vendor selection process is the technical and programmatic compatibility. The cost competiveness is secondary, and it only plays a decisive role if two vendors are selected as equally technically qualified. The OSW Team is taking this approach because it eliminates the risk of contracting with an unqualified vendor due to a selection based on a lowest cost criterion. The OSW Team is planning on identifying a second vendor as a backup should the selected vendor fail to perform. As stated earlier, the defined milestones in the contract will act as "break-points", at which time the contract can be terminated due to lack of performance if necessary. A new contract would then be established with the backup vendor to complete the scope of work. The OSW Team expects to complete the RFP process and award a vendor contract in August 2016.

\section{CONTRACT MANAGEMENT AND PERFORMANCE TRACKING}

All subcontractors engaged with the OSW project are required to comply with TMT processes, including the Software Development Process (SDP), software quality assurance processes, software configuration management processes, systems engineering processes and project management processes. The OSW team will monitor compliance throughout project execution. Non-compliances may result in termination of the contract if they are not resolved in a timely manner.

The subcontractors are bound by the agreed upon Statement of Work (SOW) and the terms of the contract, which the OSW Team and the applicable administrative team (TMTPO or IIA) will manage and enforce. As stated above, each SOW will be carefully defined to clearly specify milestones, the required deliverables for each milestone and the associated acceptance criterion; a deliverable cannot be accepted unless the acceptance criterion has been satisfied. For the OSW software development contracts, the acceptance criteria will be based on TMT functional, performance, quality and process requirements. Failure to deliver the required deliverables and/or meeting the acceptance criteria according to the contract milestone schedule may result in termination of the contract, if shortcomings are not resolved in a timely manner. Since the TMT PO and ITCC share responsibility for the OSW development activities in India, the acceptance of the deliverables are also a shared responsibility. Therefore, the OSW teams at ITCC and the TMT PO will both perform build, integration and test of the software deliverables to assess whether the software meets the acceptance criteria. Only when both the TMT PO and ITCC agree that the software deliverable meets the acceptance criteria will it be accepted.

The subcontractor payments are also tied to successful performance in addition to the accepted deliverables. TMT uses Earned Value Management (EVM) to track schedule and budget performance. At the project level, Earned Value Management metrics will be generated at the end of each software development iteration based on detailed performance measurements provided by the OSW Work Package Managers as discussed below, and provided to the TMT Project Office and ITCC leadership for review. The OSW Work Package Managers (TMT PO and ITCC) are responsible for analyzing the EVM data to make an assessment of schedule and budget performance verses expectations, justifying variances and identify issues to be resolved or opportunities for improvement. These results will be discussed with the Indian software vendor(s) during the OSW Management Team meetings and corrective actions will be devised as appropriate. If the performance issues persist, it may result in termination of the contract.

At the OSW development project level, the performance is measured by a more detailed EVM method based on completed schedule activities directly tied to the user stories and traced to the technical requirements and features. The detailed software implementation schedule is organized by the anticipated plan for iterations and their content, i.e. user 
stories allocated to iterations based on schedule priorities and an assessment of effort associated with each user story, according to the TMT software development process. To enable effective EVM, schedule updates will be based on the actual user story completion relative to plan. The completion of a user story will be verified through acceptance testing of the delivered software as discussed above. The completed user story will then be traced to the planned schedule activity and to the technical requirement and feature that it is implementing, thus maintaining traceability and ultimately ensuring that all technical requirements have been satisfied and features implemented. Once all the user stories associated with the planned schedule activity have been completed, the schedule activity will be reported as complete and the associated activity value based on the assigned effort will be earned. For CSW, the value allocation for each service and framework is shown in Table 3. This value allocation will be the basis for the earned value as each software deliverable (CSW service and framework) is accepted based on completed user stories, successful testing and software quality.

\section{CSW PLANNING AND PERFORMANCE BASELINE EXAMPLE}

The Common Software (CSW) implementation and test phase consists of the coding, unit testing, build and continuous integration, and integration testing activities for the CSW services and framework according to the TMT Software Development Process. The coding, unit testing, build and continuous integration activities will be performed by a to-beselected Indian software vendor according to the engagement and resource model defined above. Additionally, the TMT PO and ITCC will perform build, system integration and integration testing activities as part of the acceptance process following each software delivery as defined in the TMT Software Development Process. SW issues/bugs will be tracked and resolved according to the TMT Problem Tracking and Reporting Process. Performance will be measured using the EVM method discussed above.

The CSW implementation plan and EVM baseline has been developed as follows:

First, the total effort associated with each of the CSW services and framework has been estimated through a detailed decomposition of the technical requirements into user stories, and an assessment of the effort involved with each user story according to the following criteria:

Table 1. CSW effort estimation criteria

\begin{tabular}{|l|c|}
\hline User Story Size & $\begin{array}{c}\text { Estimated Effort } \\
\text { [Hours] }\end{array}$ \\
\hline Small & 8 \\
\hline Medium & 16 \\
\hline Large & 32 \\
\hline Extra Large & 48 \\
\hline Extra Extra Large & 64 \\
\hline
\end{tabular}

The total effort and relative effort was then calculated for each service and framework. Likewise, a schedule priority was assigned to each CSW service and framework, with one (1) being first priority and four (4) being lowest priority. Table 2 represents an example of effort hours, relative effort and schedule priority using the discussed method; these are not the actual effort estimates. 
Table 2. Effort and schedule priority for CSW features

\begin{tabular}{|l|r|r|r|}
\hline \multicolumn{1}{|c|}{ CSW Features } & Effort Hours & \multicolumn{1}{c|}{$\begin{array}{c}\text { Relative } \\
\text { Effort }\end{array}$} & $\begin{array}{c}\text { Schedule } \\
\text { Priority }\end{array}$ \\
\hline Time Service & 448 & 0.06 & 3 \\
\hline Locations Service & 518 & 0.06 & 1 \\
\hline Event Service & 906 & 0.11 & 1 \\
\hline Logging Service & 722 & 0.09 & 2 \\
\hline Authentication \& Authorization Service & 732 & 0.09 & 3 \\
\hline Connection \& Command Service & 1124 & 0.14 & 1 \\
\hline Configuration Service & 688 & 0.09 & 2 \\
\hline Alarm Service & 814 & 0.10 & 3 \\
\hline Database Service & 440 & 0.05 & 4 \\
\hline Framework & 1610 & 0.20 & 1 \\
\hline CSW Total Effort & 8002 & 1.00 & \\
\hline
\end{tabular}

The relative effort then was used to calculate an associated CSW feature value and "relative duration" for each CSW service and framework as follows:

- The total CSW value is based on the original estimated value in the TMT budget baseline (the total number of $\$ 500,000$ shown in Table 3 below is just an example, not the actual budget baseline), which is then allocated to each CSW feature based on the calculated relative effort

- The term "relative duration" is based on a total desired duration of the CSW implementation effort of 18 months (based on the TMT Integrated Project Schedule and CSW schedule dependencies) portioned out to each CSW feature based on their relative effort. The CSW total development duration is shown as 72 weeks, which is equivalent to 18 months assuming 4 weeks per month

Table 3. Associated value and "relative duration" for CSW features

\begin{tabular}{|l|r|r|r|r|}
\hline \multicolumn{1}{|c|}{ CSW Features } & $\begin{array}{c}\text { Effort } \\
\text { Hours }\end{array}$ & $\begin{array}{c}\text { Relative } \\
\text { Effort }\end{array}$ & $\begin{array}{c}\text { Associated } \\
\text { Value }\end{array}$ & $\begin{array}{c}\text { Relative } \\
\text { Duration } \\
\text { [Weeks] }\end{array}$ \\
\hline Time Service & 448 & 0.06 & $\$ 27,993$ & 4.0 \\
\hline Locations Service & 518 & 0.06 & $\$ 32,367$ & 4.7 \\
\hline Event Service & 906 & 0.11 & $\$ 56,611$ & 8.2 \\
\hline Logging Service & 722 & 0.09 & $\$ 45,114$ & 6.5 \\
\hline Authentication \& Authorization Service & 732 & 0.09 & $\$ 45,739$ & 6.6 \\
\hline Connection \& Command Service & 1124 & 0.14 & $\$ 70,232$ & 10.1 \\
\hline Configuration Service & 688 & 0.09 & $\$ 42,989$ & 6.2 \\
\hline Alarm Service & 814 & 0.10 & $\$ 50,862$ & 7.3 \\
\hline Database Service & 440 & 0.05 & $\$ 27,493$ & 4.0 \\
\hline Framework & 1610 & 0.20 & $\$ 100,600$ & 14.5 \\
\hline CSW Total Effort & 8002 & 1.00 & & \\
\hline CSW Total Value & & & $\$ 500,000$ & \\
\hline CSW Total Development Duration & & & & 72.0 \\
\hline
\end{tabular}


The purpose of the "relative duration" is to be able to develop a baseline schedule based on the schedule priorities defined in Table 2 and the "duration" of each CSW feature relative to the feature's effort, and thereby to be able to project planned value out over the entire 18 months of the CSW implementation project. This forms the performance baseline by which value will be earned as the contract with the Indian software vendor proceeds. A CSW implementation baseline schedule example based on this planning method and the example shown in Table 3 is shown in Figure 3.

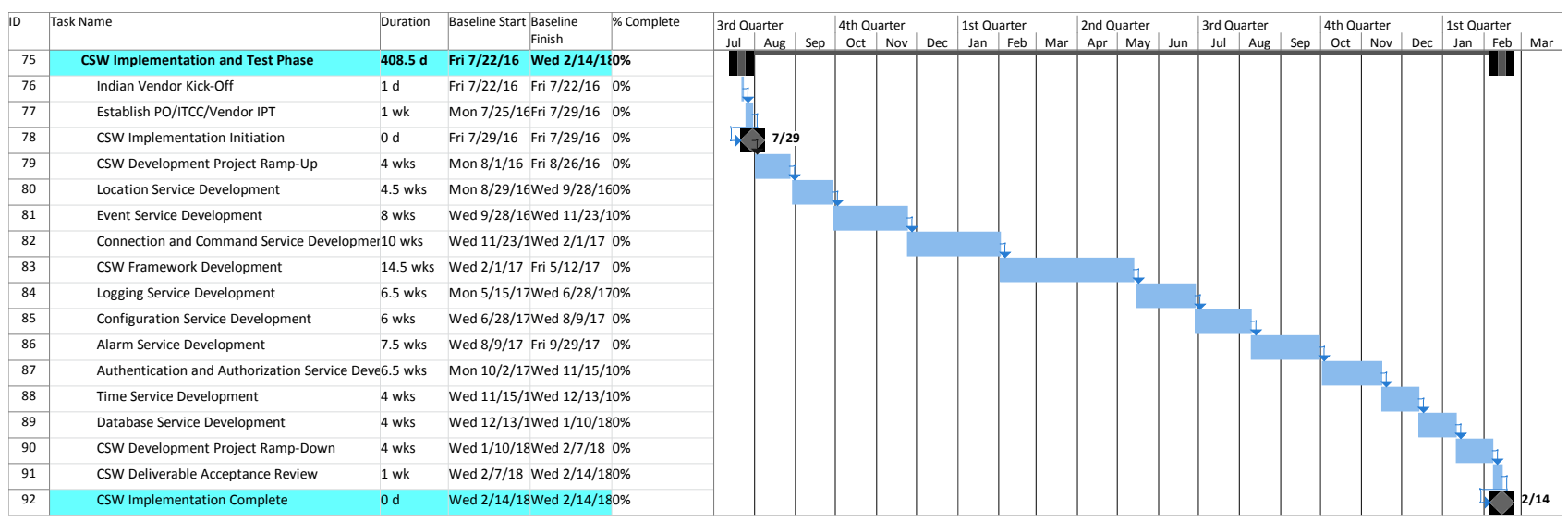

Figure 3. CSW Implementation Schedule

Once a contract with an Indian software vendor has been established, an actual schedule will be developed with the Indian software vendor to include each planned iteration with allocated user stories traceable to requirements necessary to complete each CSW feature according to the TMT established priorities, ideally within the 18 months of duration. If the actual schedule differs significantly from the planned schedule baseline based on "relative duration", the schedule baseline will be updated and the planned value adjusted. Otherwise, TMT will use the planned baseline based on "relative duration" for performance tracking and contract management as discussed in section 5.

\section{CONCLUSION}

At the time of writing this paper, all critical items in the "roadmap" are either completed or well underway as discussed in this paper. A systematic risk management approach has been adopted; the engagement and resource model, the vendor selection process, the contract management and performance tracking process, the TMT Software Development Process (SDP) and the prototyping effort are all risk mitigation strategies that have been or are in the process of being implemented.

The OSW Team, consisting of TMT PO and ITCC staff, has become a very strong team through the collaboration on CSW planning and the vendor qualification and procurement process. The OSW Team is confident in the CSW detailed design and the plans for the implementation and test phase. Likewise, the OSW Team is confident in the efficacy of the engagement and resource model, but until the model has been implemented with an Indian software vendor it is too early to declare the model a success. The OSW Team fully expects that it will require a lot of effort and persistence to make the model a success and the team is prepared to do so.

The Common Software (CSW) prototyping and final design activities were not discussed in this paper, but they are on track to finish in time for a planned Final Design Review in August 2016, which is timely for the conclusion of the vendor selection process. The OSW Team is pushing hard to get the CSW implementation and test phase kicked-off in August as planned and not to delay the TMT software subsystems dependent on CSW.

Finally, the OSW Team is very eager to work with an Indian software vendor on implementing the Agile based TMT Software Development Process (SDP) to demonstrate the benefits of continuous integration, automated testing and frequent releases, and to apply the detailed Earned Value Management (EVM) method to measure performance at the level of user stories with traceability to the CSW requirements and features. 
Successful development of the Observatory Software will play a pivotal role in TMT being able to meet its scientific objectives. We have defined and implemented a comprehensive, systematic approach to the management and execution of the OSW development project in order to ensure its success.

\section{ACKNOWLEDGEMENTS}

The TMT Project gratefully acknowledges the support of the TMT collaborating institutions. They are the Association of Canadian Universities for Research in Astronomy (ACURA), the California Institute of Technology, the University of California, the National Astronomical Observatory of Japan, the National Astronomical Observatories of China and their consortium partners, and the Department of Science and Technology of India and their supported institutes. This work was supported as well by the Gordon and Betty Moore Foundation, the Canada Foundation for Innovation, the Ontario Ministry of Research and Innovation, the National Research Council of Canada, the Natural Sciences and Engineering Research Council of Canada, the British Columbia Knowledge Development Fund, the Association of Universities for Research in Astronomy (AURA) and the U.S. National Science Foundation. 